\title{
PROBING DYNAMICAL PROCESSES IN THE PLANET-FORMING REGION WITH DUST MINERALOGY
}

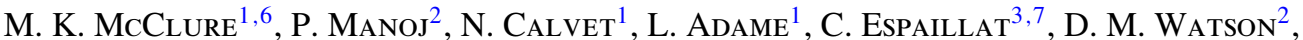 \\ B. SARGENT ${ }^{4}$, W. J. ForRest ${ }^{2}$, AND P. D'Alessio ${ }^{5}$ \\ ${ }^{1}$ Department of Astronomy, The University of Michigan, 500 Church St., 830 Dennison Bldg., Ann Arbor, MI 48109, USA; \\ melisma@umich.edu,ncalvet@umich.edu, adamel@umich.edu \\ 2 Department of Physics and Astronomy, University of Rochester, Rochester, NY 14627, USA; \\ manoj@pas.rochester.edu,dmw@pas.rochester.edu, forrest@pas.rochester.edu \\ ${ }^{3}$ Center for Astrophysics, 60 Garden Street, Cambridge, MA 02138, USA; cespaillat@ cfa.harvard.edu \\ ${ }^{4}$ Center for Imaging Science and Laboratory for Multiwavelength Astrophysics, Rochester Institute of Technology, \\ 54 Lomb Memorial Drive, Rochester, NY 14623, USA; baspci@ rit.edu \\ ${ }^{5}$ Centro de Radioastronomía y Astrofísica, Universidad Nacional Autónoma de México, 58089 Morelia, Michoacán, Mexico; p.dalessio@crya.unam.mx \\ Received 2012 September 15; accepted 2012 September 25; published 2012 October 11
}

\begin{abstract}
We present Herschel Space Observatory PACS spectra of GQ Lup, a protoplanetary disk in the Lupus star-forming region. Through spectral energy distribution fitting from $0.3 \mu \mathrm{m}$ to $1.3 \mathrm{~mm}$, we construct a self-consistent model of this system's temperature and density structures, finding that although it is $3 \mathrm{Myr}$ old, its dust has not settled to the midplane substantially. The disk has a radial gradient in both the silicate dust composition and grain size, with large amorphous grains in the upper layers of the inner disk and an enhancement of submicron, crystalline grains in the outer disk. We detect an excess of emission in the Herschel PACS B2A band near $63 \mu \mathrm{m}$ and model it with a combination of $\sim 15-70 \mu \mathrm{m}$ crystalline water ice grains with a size distribution consistent with ice recondensationenhanced grain growth and a mass fraction half of that of our solar system. The combination of crystalline water ice and silicates in the outer disk is suggestive of disk-wide heating events or planetesimal collisions. If confirmed, this would be the first detection of water ice by Herschel.
\end{abstract}

Key words: infrared: stars - protoplanetary disks - radiative transfer - stars: individual (GQ Lup)

Online-only material: color figures

\section{INTRODUCTION}

While they are a small fraction by mass of material in the interstellar medium (ISM) and the primordial disks around pre-main-sequence stars, dust grains play pivotal roles in the physical processes that shape these disks. The optical properties of the dust dictate the structure of the disk (e.g., D'Alessio et al. 2006), and spectral features can be used to probe dynamical processes such as settling, turbulence, and grain growth in the disk atmosphere (Bouwman et al. 2008; Watson et al. 2009; Turner et al. 2010).

In addition to the current disk structure, the grains' composition, structure, and size distribution record the local thermal history and kinetic conditions of the regions in which they formed. For example, silicate dust grains are at least $98 \%$ amorphous in the ISM (Kemper et al. 2004) but are found to be partly crystalline in protoplanetary disks (Bouwman et al. 2003; Sargent et al. 2009). Crystallization is highly sensitive to the specific local thermal conditions; different silicate stoichiometries form at different temperatures and pressures by thermal annealing or sublimation and recondensation (Henning \& Meeus 2011, and references therein). Both processes occur at temperatures greater than $\sim 700 \mathrm{~K}$. Nominally, this temperature is characteristic of the inner $1 \mathrm{AU}$ of a typical disk surrounding a T Tauri star (TTS). However, crystalline silicates are observed out to $40 \mathrm{AU}$ in solar system comets (Crovisier et al. 1997) and some TTS systems (Espaillat et al. 2007). Either transportation from the inner $1 \mathrm{AU}$ to the outer disk via winds, turbulent diffusion, or radial flows (Gail 2001; Ciesla 2009), or in situ formation by heating events above the $700 \mathrm{~K}$ threshold (shocks or planet

\footnotetext{
6 NSF Graduate Research Fellow.

7 NSF Astronomy and Astrophysics Postdoctoral Fellow.
}

destruction; Harker \& Desch 2002) is required to explain these results. Characterizing the mineralogical zoning of TTS disks at different radii is necessary to discriminate between these different scenarios.

Another major component of dust in disks is water ice, which may play an important role in forming planetesimals, either by helping grains stick together (Ormel et al. 2011; Kuroiwa \& Sirono 2011) or by increasing the dust to gas mass ratio at the snowline, which may induce the accumulation of grains at this location (Kretke \& Lin 2007). Although ice has been detected in absorption at NIR wavelengths in highly inclined disk systems (Pontoppidan et al. 2005), and hot water vapor has been detected in MIR Spitzer spectra (e.g., Carr \& Najita 2008), these observations probe mainly the upper layers of the disk, leaving the distribution and phase of water in the outer disk and midplane largely unknown. Recent detections of cold water vapor in TW Hya require much less water than indicated by MIR, suggesting that icy grains may settle to the midplane, leaving dry grains in the disk atmosphere (Hogerheijde et al. 2011). Identifying where and how much ice is present in TTS systems would help resolve this question.

With this motivation, we investigate GQ Lup, a 3 Myr old (Seperuelo Duarte et al. 2008) protoplanetary K7 TTS system in the Lupus molecular cloud complex with a substellar mass companion at 0.'7 separation (Neuhäuser et al. 2005) and a circumprimary disk that appears to be truncated (Dai et al. 2010). Given the range of inclination angles found for the system, $\sim 30^{\circ}-50^{\circ}$ (Broeg et al. 2007; Seperuelo Duarte et al. 2008, hereafter SD08), the separation amounts to $120-160 \mathrm{AU}$ at the distance of Lupus, 150 pc (Franco 2002). Using Herschel ${ }^{8}$

\footnotetext{
8 Herschel is an ESA space observatory with science instruments provided by European-led Principal Investigator consortia and with important participation from NASA.
} 


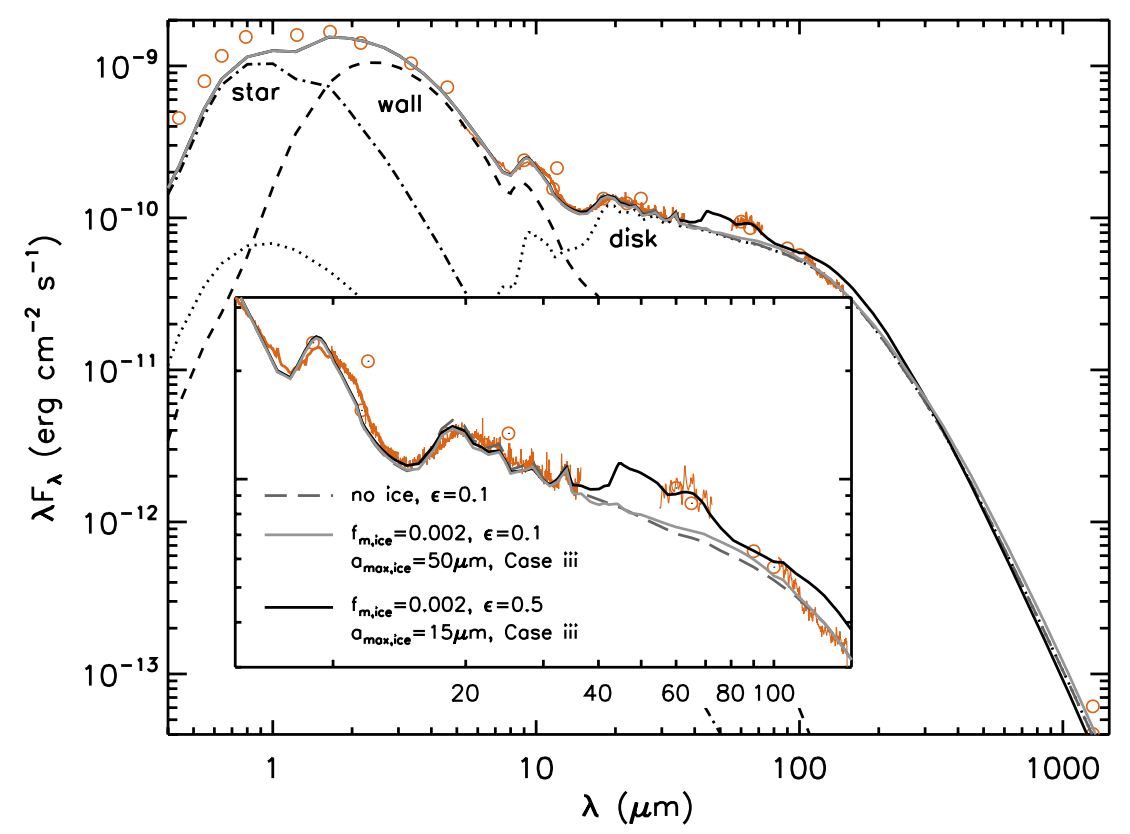

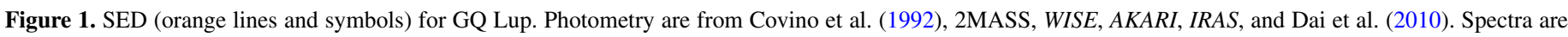

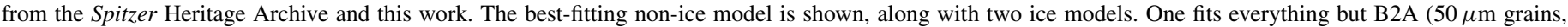

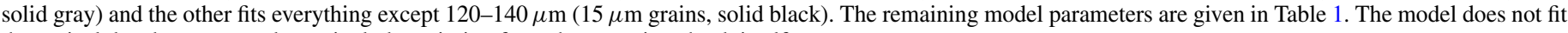
the optical data because we do not include emission from the accretion shock itself.

(A color version of this figure is available in the online journal.)

PACS, we obtained 55-145 $\mu \mathrm{m}$ spectra of GQ Lup. To characterize simultaneously the distribution of silicates and water ice in relation to the disk structure, we combined these data with archival Spitzer spectroscopy and ancillary photometry and used irradiated accretion disk models to fit the spectral energy distribution (SED) of GQ Lup.

\section{OBSERVATIONS AND DATA REDUCTION}

We observed GQ Lup using Herschel (Pilbratt et al. 2010) on 2012 January 8 (ObsID 1342238375) with PACS (Poglitsch et al. 2010) range spectroscopy modes B2A $(51-73 \mu \mathrm{m})$ and R1S $(102-145 \mu \mathrm{m})$ at Nyquist sampling $(R \sim 1500)$ and a total time of $7774 \mathrm{~s}$. The data were reduced using the standard data reduction pipeline in HIPE version 9.0 (Ott 2010). We extracted the spectra from each spaxel, confirmed that the source was point-like and well centered on the central spaxel within the pointing uncertainty of $\sim 2^{\prime \prime}$, and applied the pointspread function correction to the central spaxel spectrum. The uncertainty in PACS absolute flux calibration can be up to $30 \%$; however, GQ Lup was observed by both $I R A S$, at 60 and $100 \mu \mathrm{m}$, and $A K A R I$, at 65 and $90 \mu \mathrm{m}$. We use this photometry to confirm the absolute photometric accuracy of the PACS spectrum. The point-to-point variation of the spectrum after rebinning by a factor of 10 is $\sim 15 \%$; we assume this as our relative spectral uncertainty.

The Spitzer Infrared Spectrograph (IRS; Houck et al. 2004) low (SL, 5-14 $\mu \mathrm{m}, \lambda / \Delta \lambda=60-120$, AORID 5644032) and high (SH, 10-19 $\mu \mathrm{m}, \mathrm{LH}, 19-35 \mu \mathrm{m}, \lambda / \Delta \lambda=600$, AORID 27064576) spectral resolution data were observed on 2004 August 30 and 2008 September 2 as part of programs 172 and 50641, respectively. We reduced them with SMART (Higdon et al. 2004) in the same way as in McClure et al. (2010), with the exception that the SH/LH data were sky subtracted from off-source frames included in that AORID. We estimate the spectrophotometric uncertainty to be $\sim 5 \%$.

\section{ANALYSIS}

The SED of GQ Lup is shown in Figure 1. It has a strong excess at all infrared wavelengths, indicating the presence of a dust sublimation wall and disk. However, the disk emission drops off rapidly with increasing wavelength, consistent with the conclusion by Dai et al. (2010) that it is outwardly truncated. The Herschel B2A spectrum shows a peaked triangular shape around $63 \mu \mathrm{m}$ suggestive of the water ice feature located there. We see no evidence for a forsterite feature at $69 \mu \mathrm{m}$. In the IRS spectrum, we identify the major crystalline features by fitting a non-parametric locally weighted scatterplot smoothing baseline to the data, taking this as the "dust continuum" beneath the molecular lines, and subtracting a linear fit to regions between known crystalline silicate features to the IRS spectrum (Figure 2(a)). There are strong forsterite features at 23 and $33 \mu \mathrm{m}$, blended forsterite-enstatite features at 18 and $28 \mu \mathrm{m}$, and weak enstatite features around $11 \mu \mathrm{m}$.

To determine the composition and structure of the disk, we construct temperature and density structures using the D'Alessio et al. (2006) irradiated accretion disk models, which assume the disk is heated by stellar irradiation and viscous dissipation. Steady accretion and viscosity are parameterized through constant $\dot{M}$ and $\alpha$, respectively (Shakura \& Sunyaev 1973). The disk consists of gas and dust, the latter of which is comprised of two grain populations mixed vertically. Settling is parameterized through $\epsilon=\xi / \xi_{\text {standard }}$, where the denominator is the sum of the mass fraction of the different components relative to gas and the numerator is the mass fraction in the small dust population.

The silicate and graphite grains have size distributions $n(a)=$ $n_{0} a^{-3.5}$, where $a$ is the grain radius with limits of $0.005 \mu \mathrm{m}$ and $a_{\text {max }}$. To test whether the ice grains have grown larger than the silicate grains, we consider three size distributions: (Case i) the same power-law dependence and $a_{\max }$ as the silicate and graphite grains; (Case ii) the same power-law dependence but 

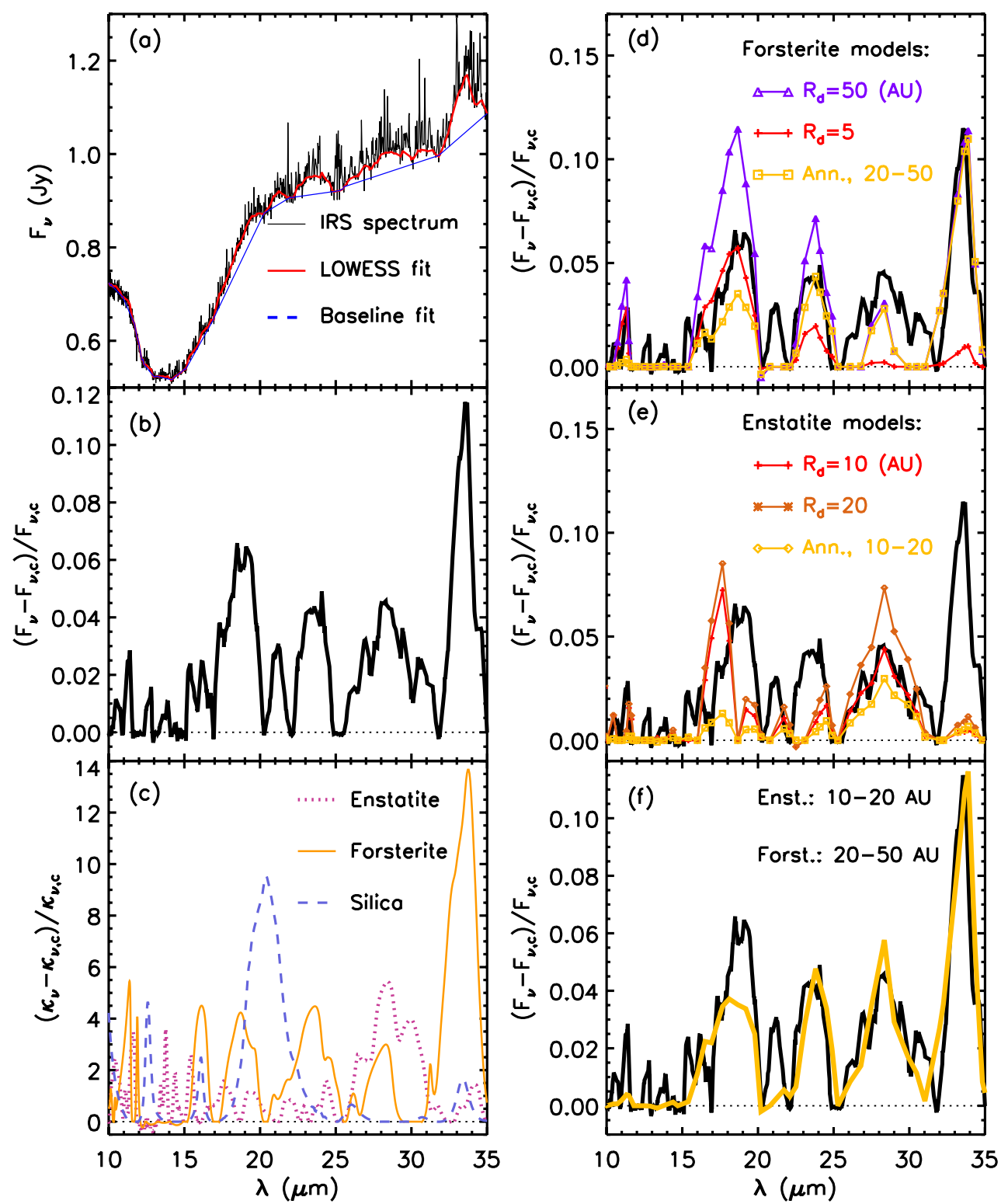

Figure 2. (a) Method for isolating crystalline silicate features in the IRS spectrum. (b) Observed crystalline features. (c) Opacities for three most common crystalline silicates. (d) Model forsterite features for fixed forsterite mass fraction and varied outer disk radius, as well as an annulus. (e) Model enstatite features with best-fitting annulus. (f) Best-fitting combination of annuli, $18 \%$ forsterite and $15 \%$ enstatite. The poor match to the $18 \mu \mathrm{m}$ region is due to fitting a linear baseline to a region that has intrinsic curvature.

(A color version of this figure is available in the online journal.)

a larger $a_{\mathrm{max}}$; and (Case iii) a power-law dependence of $n(a)=n_{0} a^{2.0}$ (e.g., Kuroiwa \& Sirono 2011). In this model, ice exists everywhere below the thermal sublimation temperature; while other processes, e.g., photodesorption, are likely to modify this snowline (Öberg et al. 2009), a self-consistent treatment is beyond the scope of this letter and reserved for future work.

We compute opacities for the graphite and water ice grains using optical constants from Draine \& Lee (1984) and Warren (1984) and Mie theory, assuming that the grains are segregated spheres (Pollack et al. 1994). Silicates are divided into amorphous and crystalline versions of two stoichiometries: olivines $\left(\mathrm{Mg}_{2-2 x} \mathrm{Fe}_{2 x} \mathrm{SiO}_{4}\right)$ and pyroxenes $\left(\mathrm{Mg}_{1-x} \mathrm{Fe}_{x} \mathrm{SiO}_{3}\right)$, where $x=\mathrm{Fe} /(\mathrm{Fe}+\mathrm{Mg})$ indicates the iron content. Opacities for the amorphous olivine and pyroxene are computed with optical constants from Dorschner et al. (1995) that have $x=0.5$ for olivine and range from 0.05 to 0.6 for pyroxene. The opacities for crystalline olivine (forsterite) and pyroxene (enstatite) are taken directly from those calculated by Sargent et al. (2009).

We implement a vertical dust sublimation wall with an atmosphere following the prescription of D'Alessio et al. (2004). Dust is present once the disk temperature drops below the dust sublimation temperature, and this radius, $R_{\text {wall }}$, defines the radial location of the beginning of the wall atmosphere, where the dust is optically thin. We allow the dust properties of the wall to vary independently of those in the disk to simulate the effects of a radial gradient in the inner disk mineralogy; in particular, we vary the iron content of the silicates in the wall, while in the disk we assume the iron content of the best-fitting amorphous olivine and pyroxene determined by Sargent et al. (2009) from comparison with disks in Taurus.

As input to the code, we assume the stellar and accretion properties within the range given by SD08 (values listed in Table 1). They measured high veiling in the system over the 
Table 1

Stellar and Model Properties

\begin{tabular}{|c|c|}
\hline Parameter & Value \\
\hline \multicolumn{2}{|r|}{ Stellar (From SD08) } \\
\hline$T_{\text {eff }}$ & $4060 \mathrm{~K}$ \\
\hline$L_{*}$ & $1.0 L_{\odot}$ \\
\hline$A_{V}$ & $0.5 \mathrm{mag}$ \\
\hline$M_{*}$ & $0.8 M_{\odot}$ \\
\hline$\dot{M}$ & $5 \times 10^{-8} M_{\odot} \mathrm{yr}^{-1}$ \\
\hline$i$ & $50^{\circ}$ \\
\hline$d$ & $155 \pm 8 \mathrm{pc}$ \\
\hline \multicolumn{2}{|r|}{ Wall } \\
\hline$T_{\text {wall }}$ & 1500 \\
\hline$R_{\text {wall }}$ & $0.19 \mathrm{AU}$ \\
\hline$z_{\text {wall }}$ & $0.03 \mathrm{AU}$ \\
\hline$a_{\max }$ & 3.0 \\
\hline Composition & $100 \%$ pyr, $40 \% \mathrm{Fe}$ \\
\hline Silicates & 0.004 \\
\hline Graphite & 0.0025 \\
\hline \multicolumn{2}{|r|}{ Disk } \\
\hline$\alpha$ & $0.01<\alpha \leqslant 0.1$ \\
\hline$\epsilon$ & $0.1<\epsilon \leqslant 0.5$ \\
\hline$R_{d}$ & $50 \mathrm{AU}$ \\
\hline \multirow[t]{2}{*}{$a_{\max }$} & $0.25 \mu \mathrm{m}$ (sil., graph.) \\
\hline & $15-70 \mu \mathrm{m}\left(\mathrm{H}_{2} \mathrm{O}\right.$ ice $)$ \\
\hline$M_{d}$ & $0.012 M_{\odot}$ \\
\hline \multirow{2}{*}{$\begin{array}{l}\text { Silicate composition } \\
\text { (radially constant) }\end{array}$} & \\
\hline & $\begin{array}{c}10 \% \text { forsterite, } 10 \% \text { enstatite, } \\
80 \% \text { pyroxene }\end{array}$ \\
\hline (annular, suggestive) & $18 \%$ forsterite, $15 \%$ enstatite in outer $20-50 \mathrm{AU}$ \\
\hline \multirow[t]{3}{*}{ Abundances } & $0.004($ sil $)$ \\
\hline & 0.0025 (graf) \\
\hline & $2 \times 10^{-3}\left(\mathrm{H}_{2} \mathrm{O}\right.$ ice $)$ \\
\hline
\end{tabular}

course of a month and estimated mass accretion rates between $10^{-8}$ and $10^{-7} M_{\odot} \mathrm{yr}^{-1}$ from comparing their observed emission lines with magnetospheric accretion models; we adopt an intermediate value of $5 \times 10^{-8} M_{\odot} \mathrm{yr}^{-1}$. The outer radius is not totally unconstrained, as the mass ratio range of the companion is $0.014-0.03$, which is too large for the circumprimary disk to radially extend over the 1:2 binary orbital resonance (Artymowicz \& Lubow 1994). Therefore, the disk can be at most $70 \mathrm{AU}$ in radius.

We create a grid of $\sim 350$ models varying $\epsilon, \alpha, a_{\max , s}$, the ice mass fraction $\left(f_{m, i}\right)$, silicate composition, and $R_{d}$ and minimize the reduced $\chi^{2}$ statistic, $\chi_{r}^{2}$, between the models and observations to determine the best fit. While there are degeneracies between $\epsilon, \alpha, f_{m, i}$ and $R_{d}$, the large wavelength coverage of our SED allows us to break some of them. For example, only $\epsilon \geqslant 0.1$ can provide enough continuum emission to fit the IRS spectrum, while $\epsilon \leqslant 0.5$ fits the slope between PACS R1S and the $1.3 \mathrm{~mm}$ photometry. Likewise, smaller disks (e.g., $R_{d} \sim 20 \mathrm{AU}$ ) underproduce the 20-35 $\mu \mathrm{m}$ absolute flux while larger disks (e.g., $R_{d} \sim 70 \mathrm{AU}$ ) overproduce the 100-140 $\mu \mathrm{m}$ flux. Once these parameters have been constrained, this implies an $\alpha$ between 0.1 and 0.01 . However, since the fit to $\alpha$ comes mainly from the submillimeter flux, which depends on the surface density, $\Sigma \propto \dot{M} / \alpha$, the estimated range of $\dot{M}$ yields $0.02 \leqslant \alpha \leqslant 0.2$ for the same fit to the SED.

Models without ice fail to reproduce either the shape or absolute flux of the PACS B2A spectrum; in Figure 3(a) we show this model and how the SED changes when ice with the distributions given by the previously defined size distributions Cases (i), (ii), and (iii) is added. Case (i) lowers the flux too much over the Spitzer range and fails to produce enough flux over B2A. Case (ii) produces only a slight increase in the flux between 55 and $70 \mu \mathrm{m}$. With Case (iii), we are able to produce enough flux over both the IRS and PACS ranges. For Case (iii), the B2A and R1S spectra are better reproduced by different $a_{\max , i}, 15 \mu \mathrm{m}$ and 50-70 $\mu \mathrm{m}$, respectively (Figure 3(b)). The mass fraction of ice that produces the best fit to the data for this disk structure is $2 \times 10^{-3}$ with respect to gas, or $\sim 25 \%$ of the total dust content, which is half of the solar composition (Pollack et al. 1994). Although our model includes ice at this abundance in the midplane as well, because the disk has so little dust settling, we would require spatially resolved submillimeter photometry to test whether the midplane ice abundance differs from that of the upper layers.

The best-fitting wall has an $a_{\max }$ of $3 \mu \mathrm{m}$ and is comprised of $100 \%$ glassy pyroxene with $\mathrm{Fe} /(\mathrm{Fe}+\mathrm{Mg})=0.4$. With the assumption of a radially uniform disk composition, the bestfitting models require silicate grains with $a_{\max }=0.25 \mu \mathrm{m}$ and a mixture of $80 \%$ amorphous silicates, of which $5 \%$ are olivine and $75 \%$ pyroxene $(\mathrm{Fe} /(\mathrm{Fe}+\mathrm{Mg})=0.2)$, and $20 \%$ crystalline silicates, evenly split between forsterite and enstatite. Although our model does not yet include radial composition gradients, we can still use it to probe the location of the crystalline silicates. We compute a grid of theoretical disks truncated outwardly at $5 \mathrm{AU}$ intervals from $50 \mathrm{AU}$ to $5 \mathrm{AU}$ for compositions of pyroxene-forsterite and pyroxene-enstatite, allowing the individual crystalline silicate fractions to vary between 5\% and 30\%. By isolating the model forsterite features in the same manner as for the IRS spectrum, we see that the models with sufficient forsterite abundance to fit the $33 \mu \mathrm{m}$ feature produce too much flux in the $23 \mu \mathrm{m}$ features (Figure 2) and vice versa, indicating that forsterite is not uniformly distributed throughout the disk.

To compare the data with the emission from an annular region of the disk, we subtract the emission of the other radii from the $50 \mathrm{AU}$ disk and compare the ratio of the 23 to $33 \mu \mathrm{m}$ feature to determine which emitting annulus is the best match to our data. Forsterite distributed between 20 and $50 \mathrm{AU}$ fits the relative fluxes best and implies a forsterite silicate fraction of $\sim 18 \%$ in this region. A match to the observed enstatite emission of an annulus of $10-20 \mathrm{AU}$ with a fraction of $15 \%$ is obtained in the same way. We note that the non-detection of the $69 \mu \mathrm{m}$ forsterite feature in the PACS spectrum places an upper limit of $90 \%$ on the forsterite fraction in the outer disk upper layers. To cross-check our results, we perform a two-temperature spectral decomposition (e.g., Sargent et al. 2009) on the IRS spectrum. Its results confirm that the inner disk is dominated by large, amorphous pyroxene grains with no crystalline grains detected at $>1 \sigma$. The outer disk, however, contains small pyroxene grains with forsterite and enstatite present at $3 \sigma$ and $2 \sigma$, respectively. The resulting system properties, assuming radially constant dust abundances in the disk, are listed in Table 1. The final best fit has $\chi_{r}^{2}=1.2$, and is displayed in Figure 1 .

\section{DISCUSSION AND CONCLUSIONS}

\subsection{An Iron-rich and Turbulent Inner Disk?}

The radius of the wall is proportional to the ratio of a grain's opacity weighted by the stellar and local temperature and the assumed dust sublimation temperature. Of the grains we tested, grains with $x=0.4$ (40\% Fe content) had the largest opacity 


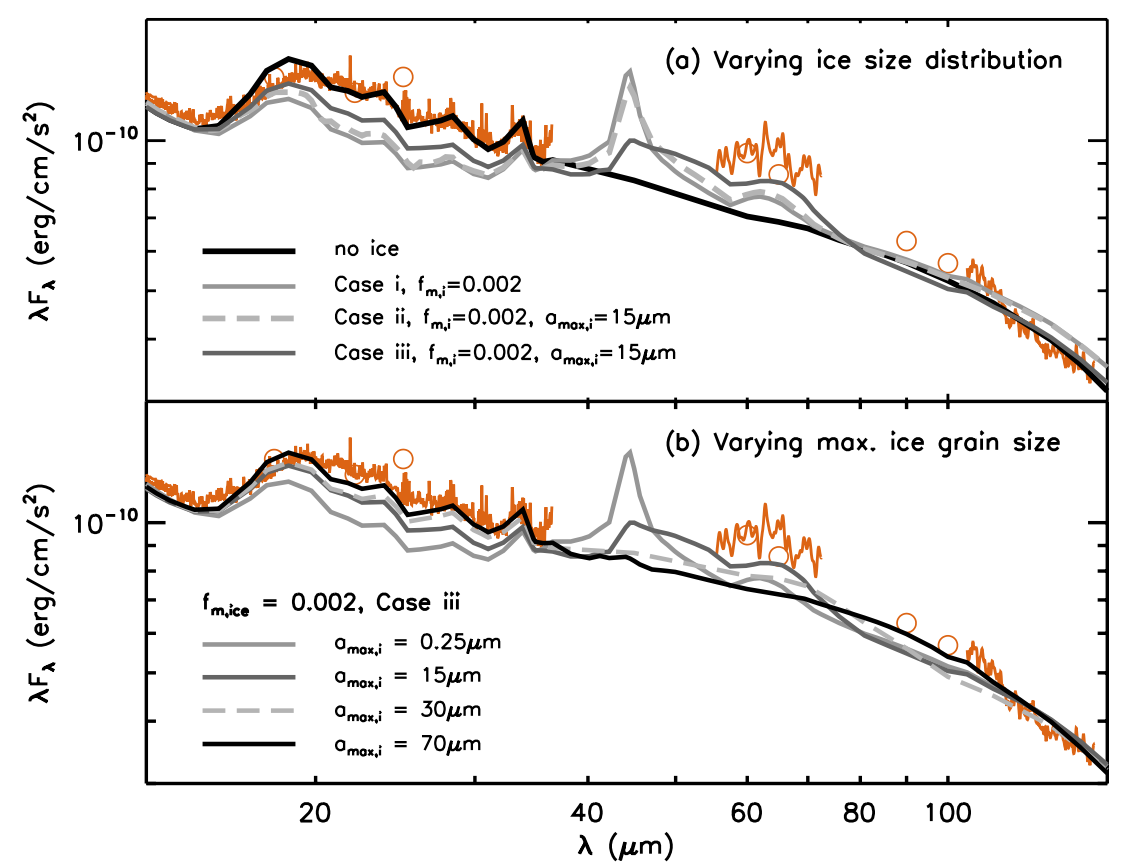

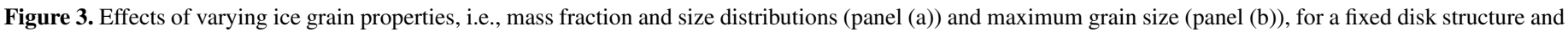
silicate/graphite dust properties.

(A color version of this figure is available in the online journal.)

ratio, so they absorb heat more efficiently than they emit it and achieve the dust sublimation temperature at larger radii than grains with less iron. For a wall whose height is given in a fixed number of gas pressure scale heights, the solid angle to the observer is then larger, and therefore the peak observed flux is greater. The high iron content of our inner disk is consistent with the $\mathrm{Fe} / \mathrm{Mg} \sim 1$ ratio found, on average, for pre-solar silicate grains in meteorites (Nguyen et al. 2010). However, this work does not explore the possibility that other near-infrared continuum sources that might be expected in the inner disk, e.g., metallic iron which represents $60 \%$ of Mercury's total mass, could be present in the wall. The large maximum grain size of the wall, taken in conjunction with the high $\dot{M}$ and little settling, is indicative of the turbulent enrichment of the inner disk atmosphere with large grains seen in other systems by Sicilia-Aguilar et al. (2007). It also suggests that there is a radial gradient in the maximum grain size of the upper layers, as the outer disk is best fit with $0.25 \mu \mathrm{m}$ grains.

\subsection{Ice-enhanced Grain Growth}

Although the silicate and graphite grains are small in the outer disk, we can reproduce better the broader shape and flux of the SED from 50 to $70 \mu \mathrm{m}$ with a population of larger ice grains in the upper disk layers (Figure 3(b)). The $63 \mu \mathrm{m}$ peak is best fit by an $a_{\max }$ of $15 \mu \mathrm{m}$ and a larger $\epsilon$, while a range of $a_{\max }$ between 20 and $50 \mu \mathrm{m}$ can provide the bulk of the excess flux between 30 and $100 \mu \mathrm{m}$. The fact that we need ice grains two orders of magnitude larger than the silicate grains and with a different grain size distribution power law is consistent with simulations for ice enhancement of grain growth that take recondensation into account (Kuroiwa \& Sirono 2011), if we assume that their ice-coated dust grain opacities can be approximated by our segregated ice grain opacities when $a_{\max , i}$ is large. The better fit to the $120 \mu \mathrm{m}$ region provided by the $50 \mu \mathrm{m}$ ice grains may indicate either a radial or a vertical gradient in the ice grain size, which we do not yet implement in this version of our model.
We can think of two potential explanations for why we might see an ice emission feature in this system and not (to the best of our knowledge) in other systems. First, the disk of GQ Lup is highly unsettled $(\epsilon=0.1-0.5)$. This implies mixing of grain populations between the midplane and upper layers. If icy grains are inclined to settle to the midplane or are formed there, we may see them because they are being turbulently lifted back to the disk surface. Additionally, the lack of settling means that the location of the $A_{V}=3$ isocontour (which defines where ice ceases to be photodesorbed; Gorti \& Hollenbach 2008) is at a large enough vertical height in the disk that some ice should remain on the grains above the $\tau=1$ surface for $63 \mu \mathrm{m}$ emission. Alternatively, if there are planetesimals in the disk, they would not appear to be large enough to clear a gap on the order of tens of AU, as we do not see evidence for one in the SED (although we note that this does not constrain smaller gaps; Espaillat et al. 2010). The presence of a massive, gaseous disk of $R_{d}=30-100 \mathrm{AU}$ at the onset of the formation of Jovian planets has been simulated to scatter smaller planetesimals in the disk, populating high inclination orbits at $\sim 40 \mathrm{AU}$ (Kretke et al. 2012). If some of these icy planetesimals were to collide, it could generate a reservoir of icy grains in the upper layers (e.g., Lisse et al. 2012).

\subsection{Evidence for Localized Heating in the Outer Disk?}

The presence of the $63 \mu \mathrm{m}$ feature is also of particular interest, as it appears only in crystalline ice, and the crystallization temperature of initially amorphous water ice is typically $>130 \mathrm{~K}$ (Baragiola 2003). Although the densities in our disk structure imply an ice sublimation temperature of at most $120 \mathrm{~K}$ (Pollack et al. 1994), it has been shown that ice deposited on grains at $160 \mathrm{~K}$ and subsequently cooled to $14 \mathrm{~K}$ maintains the $63 \mu \mathrm{m}$ opacity peak (Moore et al. 2001). This implies that GQ Lup has experienced significant heating and cooling events in the outer disk. 
Localized heating could also explain the annular crystalline distribution suggested by the last analysis in Section 3. This distribution is not indicative of transport models, which we would expect to yield monotonically decreasing abundances, like those found by van Boekel et al. (2004) for some Herbig AeBe stars. However, there are in situ mechanisms that could form crystals at tens of AU. For a typical TTS, Harker \& Desch (2002) found that shocks from gravitational instabilities can anneal dust between 5 and $10 \mathrm{AU}$, assuming an annealing temperature around $1200 \mathrm{~K}$. Crystalline dust could also be produced through collisions of parent bodies, as seen in older stars (e.g., Lisse et al. 2012). Given the 3 Myr age of GQ Lup, it is not unfeasible that KBO-size planetesimals could have formed in the outer disk.

In conclusion, the mineralogical and disk structure properties of GQ Lup make it an interesting object with which to test theories of planetesimal formation. The grains have grown to micron size in the inner disk, while the outer disk is highly unsettled and shows signs of water ice in its PACS spectrum at sizes consistent with models of ice-enhanced grain growth. The presence of crystalline ice and silicates at temperatures below their crystallization temperature and in annular distributions is indicative of a periodic in situ heating mechanism. Follow-up studies should be conducted to confirm the disk size, resolve any radial structure at larger radii than those probed by our spectra, and probe the midplane grain size distributions.

M.K.M., N.C. and L.A., C.E., and P.D. acknowledge support from these sources, respectively: NSF GRFP (grant No. DGE 0718128), NASA Origins (grant No. NNX08AH94G), NSF (award No. 0901947), and PAPIIT UNAM.

\section{REFERENCES}

Artymowicz, P., \& Lubow, S. H. 1994, ApJ, 421, 651

Baragiola, R. A. 2003, Planet. Space Sci., 51, 953

Bouwman, J., de Koter, A., Dominik, C., \& Waters, L. B. F. M. 2003, A\&A, 401, 577

Bouwman, J., Henning, T., Hillenbrand, L. A., et al. 2008, ApJ, 683, 479

Broeg, C., Schmidt, T. O. B., Guenther, E., et al. 2007, A\&A, 468, 1039

Carr, J. S., \& Najita, J. R. 2008, Science, 319, 1504

Ciesla, F. J. 2009, Meteorit. Planet. Sci., 44, 1663

Covino, E., Terranegra, L., Franchini, M., Chavarria-K., C., \& Stalio, R. 1992, A\&AS, 94, 273

Crovisier, J., Leech, K., Bockelee-Morvan, D., et al. 1997, Science, 275, 1904

Dai, Y., Wilner, D. J., Andrews, S. M., \& Ohashi, N. 2010, AJ, 139, 626
D’Alessio, P., Calvet, N., Hartmann, L., Franco-Hernández, R., \& Servín, H. 2006, ApJ, 638, 314

D’ Alessio, P., Calvet, N., Hartmann, L., Muzerolle, J., \& Sitko, M. 2004, in IAU Symp. 221, Star Formation at High Angular Resolution, ed. M. G. Burton, R. Jayawardhana, \& T. L. Bourke (Cambridge: Cambridge Univ. Press), 403

Dorschner, J., Begemann, B., Henning, T., Jaeger, C., \& Mutschke, H. 1995, A\&A, 300, 503

Draine, B. T., \& Lee, H. M. 1984, ApJ, 285, 89

Espaillat, C., Calvet, N., D’Alessio, P., et al. 2007, ApJ, 670, L135

Espaillat, C., D’Alessio, P., Hernández, J., et al. 2010, ApJ, 717, 441

Franco, G. A. P. 2002, MNRAS, 331, 474

Gail, H.-P. 2001, A\&A, 378, 192

Gorti, U., \& Hollenbach, D. 2008, ApJ, 683, 287

Harker, D. E., \& Desch, S. J. 2002, ApJ, 565, L109

Henning, T., \& Meeus, G. 2011, in Physical Processes in Circumstellar Disks around Young Stars, ed. P. J. V. Garcia (Chicago, IL: Chicago Univ. Press), 114

Higdon, S. J. U., Devost, D., Higdon, J. L., et al. 2004, PASP, 116, 975

Hogerheijde, M. R., Bergin, E. A., Brinch, C., et al. 2011, Science, 334, 338

Houck, J. R., Roellig, T. L., van Cleve, J., et al. 2004, ApJS, 154, 18

Kemper, F., Vriend, W. J., \& Tielens, A. G. G. M. 2004, ApJ, 609, 826

Kretke, K. A., Levison, H. F., Buie, M. W., \& Morbidelli, A. 2012, AJ, 143,91

Kretke, K. A., \& Lin, D. N. C. 2007, ApJ, 664, L55

Kuroiwa, T., \& Sirono, S.-i. 2011, ApJ, 739, 18

Lisse, C. M., Wyatt, M. C., Chen, C. H., et al. 2012, ApJ, 747, 93

McClure, M. K., Furlan, E., Manoj, P., et al. 2010, ApJS, 188, 75

Moore, M. H., Hudson, R. L., \& Gerakines, P. A. 2001, Spectrochimica Acta, 57,843

Neuhäuser, R., Guenther, E. W., Wuchterl, G., et al. 2005, A\&A, 435, L13

Nguyen, A. N., Nittler, L. R., Stadermann, F. J., Stroud, R. M., \& Alexander, C. M. O. 2010, ApJ, 719, 166

Öberg, K. I., Linnartz, H., Visser, R., \& van Dishoeck, E. F. 2009, ApJ, 693, 1209

Ormel, C. W., Min, M., Tielens, A. G. G. M., Dominik, C., \& Paszun, D. 2011, A\&A, 532, A43

Ott, S. 2010, in ASP Conf. Ser. 434, Astronomical Data Analysis Software and Systems XIX, ed. Y. Mizumoto, K.-I. Morita, \& M. Ohishi (San Francisco, CA: ASP), 139

Pilbratt, G. L., Riedinger, J. R., Passvogel, T., et al. 2010, A\&A, 518, L1

Poglitsch, A., Waelkens, C., Geis, N., et al. 2010, A\&A, 518, L2

Pollack, J. B., Hollenbach, D., Beckwith, S., et al. 1994, ApJ, 421, 615

Pontoppidan, K. M., Dullemond, C. P., van Dishoeck, E. F., et al. 2005, ApJ, 622,463

Sargent, B. A., Forrest, W. J., Tayrien, C., et al. 2009, ApJS, 182, 477

Seperuelo Duarte, E., Alencar, S. H. P., Batalha, C., \& Lopes, D. 2008, A\&A, 489,349

Shakura, N. I., \& Sunyaev, R. A. 1973, A\&A, 24, 337

Sicilia-Aguilar, A., Hartmann, L. W., Watson, D., et al. 2007, ApJ, 659, 1637

Turner, N. J., Carballido, A., \& Sano, T. 2010, ApJ, 708, 188

van Boekel, R., Min, M., Leinert, C., et al. 2004, Nature, 432, 479

Warren, S. G. 1984, Appl. Opt., 23, 1206

Watson, D. M., Leisenring, J. M., Furlan, E., et al. 2009, ApJS, 180, 84 